IPM/P-2006/038

\title{
New Attractors, Entropy Function and Black Hole Partition Function
}

\author{
Mohsen Alishahiha1 and Hajar Ebrahim² \\ Institute for Studies in Theoretical Physics and Mathematics (IPM) \\ P.O. Box 19395-5531, Tehran, Iran
}

\begin{abstract}
By making use of the entropy function formalism we study the generalized attractor equations in the four dimensional $\mathcal{N}=2$ supergravity in presence of higher order corrections. This result might be used to understand a possible ensemble one could associate to an extremal black hole.
\end{abstract}

\footnotetext{
${ }^{1}$ alishah@theory.ipm.ac.ir

2ebrahim@theory.ipm.ac.ir
} 


\section{Introduction}

Recently the black hole attractor mechanism has attracted a lot of attention. This is mainly because of the recent developments in the connection between the partition function of four dimensional BPS black holes and partition function of topological strings [1].

According to the attractor mechanism the values of the moduli scalar fields at the horizon are entirely determined by the charges of the black hole regardless of their asymptotic values. Originally this special behavior has been discovered in the context of BPS extremal black holes in four dimensional $\mathcal{N}=2$ supergravity with unbroken supersymmetry in $[2-5]$. Later on it was shown that the attractor mechanism can also work for non-BPS extremal black holes [6-8]. In particular new algebraic attractor equations describing both BPS and non-BPS solutions have been introduced in [9] (for further discussions see [10-13]).

More recently attractor mechanism has been studied in non-supersymmetric extremal black holes [14] (see also [15]). In fact a similar structure to supersymmetric extremal black holes appears in non-supersymmetric cases. Namely the true attractive points correspond to the critical points of the black hole effective potential which make the potential minimum. Moreover the entropy of these non-supersymmetric extremal black holes is given by the value of the effective potential at the extremum and therefore due to the attractor behavior, it is given by the charges of the black hole.

On the other hand a general method for computing the entropy of spherically symmetric extremal black holes in a theory of gravity coupled to gauge fields and scalar fields has been developed in [16]. In this method one can obtain the entropy of the extremal black hole just by using its near horizon field configuration, assuming the existence of the full black hole solution. To be precise let us consider an extremal $d$-dimensional black hole whose near horizon geometry is $A d S_{2} \times S^{d-2}$ and carries electric and magnetic charges. There are also several scalar fields in the theory. One then defines the entropy function as the Legendre transform of the Lagrangian density integrated over angular variables, with respect to the value of the electric field strength at the horizon. Extremizing the entropy function with respect to near horizon variables will result in a set of algebraic equations for these parameters. The entropy of these black holes is given by the value of the entropy function evaluated at the extremum 3 .

It is shown that the entropy function is actually proportional to the effective potential of non-supersymmetric extremal black holes [19]. In this sense the entropy function is given directly in terms of the prepotential in the supersymmetric case. We will come back to this point in section 4 .

It is worth noting that due to the algebraic nature of the equations, higher

\footnotetext{
${ }^{3}$ We note that a similar function has also been found in the supersymmetric case in $[17,18]$. Although in the supersymmetric case it is not called entropy function, it has precisely the same properties.
} 
derivative corrections to the action and entropy can be obtained more simply in this formalism. Indeed this method has been used to compute corrections to the entropy of the different extremal black holes in [20-24].

Although the entropy function has mainly been used in non-supersymmetric theories, this formalism has also been applied to $\mathcal{N}=2$ supergravity theories in [25] where the authors have shown that the BPS attractor equations can be obtained by extremizing the entropy function with respect to the black hole charges. It is one of the aims of this paper to study the generalized attractor equations using the entropy function which can be applied to supersymmetric as well as non-supersymmetric theories. Being simple, this formalism can be used to find the attractor equations in the presence of higher order corrections. The supersymmetric attractor equations in the presence of higher order corrections have been studied in [26].

Having had the attractor equations which come out from the equations of motion, one may ask if the entropy function mechanism is just a technical method. In other words we would like to understand the physical interpretation (if any) of the entropy function which is essentially equivalent to the Wald formula for the entropy. In order to address this question we will follow the recent works on connection between topological string theories and black hole partition function [1] where the authors have proposed a mixed ensemble for the extremal black hole of four dimensional supergravity obtained by compactification of type IIA on Calabi-Yau 3 -fold. In particular we would like to compare the entropy function formalism with the structure used in [1] (see also [27-31]).

This paper is organized as follows. In section 2 we rederive the generalized attractor equations in $\mathcal{N}=2$ supergravity theories by extremizing the effective potential. In section 3 we show how the generalized attractor equations can be obtained in entropy function formalism where higher order corrections can also be taken into account. In section 4 we will study the partition function of the extremal black holes in the context of entropy function formalism following OSV conjecture. The last section is devoted to discussions.

\section{Generalized attractor equations}

In this section we rederive generalized attractor equations in $\mathcal{N}=2$ four dimensional supergravity coupled to $n$ vector multiplets. These equations have recently been studied in [10-13]. Using these equations one can study BPS and non-BPS attractive points in the same way. The aim of this section is to present a direct derivation of these generalized attractor equations by minimizing the leading order effective potential.

Consider $\mathcal{N}=2$ four dimensional supergravity coupled to $n$ vector multiplets. To study these theories it is useful to work within the framework of the special geometry. A special Kähler manifold can be constructed by a $2 n+2$ dimensional 
flat symplectic bundle over a Kähler manifold with a symplectic section defined by

$$
\Pi=\left(L^{I}, M_{I}\right), \quad I=0, . ., n,
$$

subject to a constraint $i\left(\bar{L}^{I} M_{I}-L^{I} \bar{M}_{I}\right)=1$. $L^{I}$ and $M_{I}$ depend on scalar fields $t$ and $\bar{t}$ which parameterize the moduli space. They are also covariantly holomorphic which means $D_{\bar{i}} \Pi=\left(\partial_{\bar{i}}-\frac{1}{2} K_{\bar{i}}\right) \Pi=0$. Here $K$ is the Kähler potential.

Introducing a symplectic charge $\left(q_{I}, p^{I}\right)$ one can define a covariantly holomorphic central charge as

$$
Z(t, \bar{t}, p, q) \equiv\left(L^{I} q_{I}-M_{I} p^{I}\right),
$$

which satisfies $D_{\bar{i}} Z=D_{i} \bar{Z}=0$. From four dimensional supergravity point of view one may identify this with the charge of the graviphoton. On the other hand one may identify the $\left(q_{I}, p^{I}\right)$ with the charges of a black hole solution in this four dimensional supergravity whose effective potential is given by

$$
V_{\text {eff }}=|Z|^{2}+\left|D_{i} Z\right|^{2}
$$

which is symplectic invariant. It is known that the attractor equations can be obtained by extremizing this potential $[6,32]$.

The extremization of the effective potential will give the following condition

$$
2\left(D_{i} Z\right) \bar{Z}+G^{j \bar{k}} D_{i} D_{j} Z \bar{D}_{\bar{k}} \bar{Z}=0,
$$

which can be solved to find the attractor points. One of its solutions is given by

$$
D_{i} Z=\bar{D}_{\bar{i}} \bar{Z}=0
$$

which leads to the supersymmetric attractor equations as follows

$$
p^{I}=i\left(\bar{Z} L^{I}-Z \bar{L}^{I}\right), \quad q_{I}=i\left(\bar{Z} M_{I}-Z \bar{M}_{I}\right) .
$$

One may also relax the supersymmetric condition $D_{i} Z=0$ and look for a general solution of equation (2.4) which could lead to non-supersymmetric equations as well. To do this let us start with the conjugate form of the equation (2.4). Using the definition of $Z$ the first term in the equation (2.4) reads

$$
2 Z \bar{D}_{\bar{i}} \bar{Z}=2 Z\left(D_{\bar{i}} \bar{L}^{I} q_{I}-D_{\bar{i}} \bar{M}_{I} p^{I}\right) .
$$

One can replace $D_{\bar{i}} \bar{M}_{I}$ with $\mathcal{N}_{I J} D_{\bar{i}} \bar{L}^{J}$, where $\mathcal{N}_{I J}$ is a complex symmetric $(n+1) \times$ $(n+1)$ matrix such that $D_{\bar{i}} \bar{M}_{I}=\mathcal{N}_{I J} D_{\bar{i}} \bar{L}^{J}$. Now contracting both sides of the equation with $G^{i \bar{i}} D_{i} L^{K}$ and using the identity

$$
D_{i} L^{I} G^{i \bar{i}} D_{\bar{i}} \bar{L}^{J}=-\frac{1}{2} \operatorname{Im}\left(\mathcal{N}^{-1}\right)^{I J}-\bar{L}^{I} L^{J},
$$

we will obtain the following relation for the charges $p^{I}$

$$
p^{I}=i\left[-2 Z \bar{L}^{I}+\frac{G^{i \bar{j}} G^{l \bar{k}} \bar{D}_{\bar{j}} \bar{D}_{\bar{k}} \bar{Z} D_{l} Z D_{i} L^{I}}{Z}-\left[\operatorname{Im}\left(\mathcal{N}^{-1}\right) q+\left(\operatorname{Re} \mathcal{N} \operatorname{Im} \mathcal{N}^{-1}\right) p\right]^{I}\right]
$$


To get the other charges $q_{I}$ we first multiply the above equation by $\left(\overline{\mathcal{N}}^{-1}\right)^{I L}$ and then taking into account that $\mathcal{N}_{I J}\left(\mathcal{N}^{-1}\right)^{J L}=\delta_{I}^{L}$, we arrive at

$$
\begin{aligned}
q_{I} & =i\left[-2 Z \bar{M}_{I}+\frac{G^{i \bar{j}} G^{l \bar{k}} \bar{D}_{\bar{j}} \bar{D}_{\bar{k}} \bar{Z} D_{l} Z D_{i} M_{I}}{Z}\right. \\
& \left.+\left[\left(\operatorname{Re} \mathcal{N} \operatorname{Im} \mathcal{N}^{-1} \operatorname{Re} \mathcal{N}+\operatorname{Im} \mathcal{N}\right) p-\left(\operatorname{Im} \mathcal{N}^{-1} \operatorname{Re} \mathcal{N}\right) q\right]_{I}\right]
\end{aligned}
$$

Now taking the imaginary parts of the above relations we can obtain the generalized attractor equations which are

$$
\begin{aligned}
q_{I} & =2 \operatorname{Im}\left[Z \bar{M}_{I}-\frac{G^{i \bar{j}} G^{l \bar{k}} \bar{D}_{\bar{j}} \bar{D}_{\bar{k}} \bar{Z} D_{l} Z}{2 Z} D_{i} M_{I}\right], \\
p^{I} & =2 \operatorname{Im}\left[Z \bar{L}^{I}-\frac{G^{i \bar{j}} G^{l \bar{k}} \bar{D}_{\bar{j}} \bar{D}_{\bar{k}} \bar{Z} D_{l} Z}{2 Z} D_{i} L^{I}\right] .
\end{aligned}
$$

Here we assume that $Z \neq 0$. This is the general form of the attractor equations in leading order which are valid for both supersymmetric and non-supersymmetric cases. In particular setting $D_{i} Z=0$ one gets the supersymmetric equations. They have exactly the same form as those in [12].

\section{Generalized attractor equations from entropy function}

In this section we shall study the attractor equations using the entropy function formalism [16]. It is important to note that the attractor equations presented in the previous section are valid only in leading order. In fact taking into account higher order corrections to the action one needs to minimize the corrected effective potential. The supersymmetric attractor equations in presence of higher order corrections have been studied in [26].

We note, however, that using the entropy function one may easily handle the higher order corrections in the same way as the leading order both for supersymmetric and non-supersymmetric cases. Actually it is the aim of this section to derive the generalized attractor equations for $\mathcal{N}=2$ supergravity in four dimensions when the higher order corrections are also taken into account.

\subsection{General formalism}

Let us first review the minimum ingredients we need to write the off-shell form of $\mathcal{N}=2$ supergravity action in four dimensions (see for example [33-35]). In what follows we use the notation of [36]. To study this theory it is useful to start with superconformal theory and then we can fix the gauge to get the supergravity 
theory we are interested in. The representation of the corresponding superconformal algebra contains Weyl, vector and non-linear multiplets.

Since we are interested in the off-shell representation, these multiplets contain dynamical bosonic fields, the corresponding fermionic superpartners and nondynamical fields. The dynamical bosonic fields of the theory are $(N+1)$ complex scalars $X^{I}$ with $0 \leq I \leq N$, metric $G_{\mu \nu}$ and $(N+1)$ gauge fields $A_{\mu}^{I}$. The nondynamical fields of the multiplets are a complex anti-self-dual antisymmetric tensor field $T_{\mu \nu}^{-}$, a real scalar field $D$, a $U(1)$ gauge field $\mathcal{A}_{\mu}$, an $S U(2)$ gauge field $\mathcal{V}_{j \mu}^{i}$, a vector field $V_{\mu}$, a set of $S U(2)$ triplet scalar fields $Y_{i j}^{I}$, an $S U(2)$ triplet scalar field $M_{i j}$ and scalar field $\Phi_{i}^{\alpha}$ which transform as a fundamental of both the gauge $S U(2)$ and global $S U(2)$ symmetries. Here $i, j=1,2$ are $S U(2)$ indices which are raised and lowered by the anti-symmetric tensor $\epsilon^{i j}$ and $\epsilon_{i j}$. There are also fermionic fields which are not presented here.

In this formulation the action involving these fields can be written in terms of the prepotential $F\left(X^{I}, \hat{A}\right)$, which is a homogeneous function of the complex scalars $X^{I}$ and the composite auxiliary field $\hat{A}=T^{-\mu \nu} T_{\mu \nu}^{-}$such that

$$
F\left(\lambda X^{I}, \lambda^{2} \hat{A}\right)=\lambda^{2} F\left(X^{I}, \hat{A}\right)
$$

In terms of the prepotential, defining

$$
F_{I}=\frac{\partial F}{\partial X^{I}}, \quad F_{\hat{A}}=\frac{\partial F}{\partial \hat{A}}, \quad F_{I J}=\frac{\partial^{2} F}{\partial X^{I} \partial X^{J}}, \quad F_{I \hat{A}}=\frac{\partial^{2} F}{\partial X^{I} \partial \hat{A}}, \quad F_{\hat{A} \hat{A}}=\frac{\partial^{2} F}{\partial \hat{A} \partial \hat{A}},
$$

the bosonic part of the Lagrangian is given by ( see equation (3.111) of [36])

$$
\begin{aligned}
8 \pi \mathcal{L} & =-\frac{i}{2}\left(X^{I} \bar{F}_{I}-\bar{X}^{I} F_{I}\right) R+\left[i\left(\partial_{\mu} F_{I}+i \mathcal{A}_{\mu} F_{I}\right)\left(\partial^{\mu} \bar{X}^{I}-i \mathcal{A}^{\mu} \bar{X}^{I}\right)+\frac{i}{32} \bar{F} \hat{A}\right. \\
& +\frac{i}{4} F_{I J} A_{\mu \nu}^{I} A^{J \mu \nu}+\frac{i}{8} \bar{F}_{I} A_{\mu \nu}^{I} T^{-\mu \nu}+\frac{i}{2} \hat{F}_{\mu \nu}^{-} F_{I \hat{A}} A^{I \mu \nu}-\frac{i}{8} F_{I J} Y_{i j}^{I} Y^{J i j}+\frac{i}{2} F_{\hat{A}} \hat{C} \\
& \left.-\frac{i}{8} F_{\hat{A} \hat{A}}\left(\hat{B}_{i j} \hat{B}^{i j}-2 \hat{F}_{\mu \nu}^{-} \hat{F}^{-\mu \nu}\right)-\frac{i}{4} \hat{B}_{i j} F_{I \hat{A}} Y^{I i j}+h . c .\right] \\
& -i\left(X^{I} \bar{F}_{I}-\bar{X}^{I} F_{I}\right)\left(\nabla^{\mu} V_{\mu}-\frac{1}{2} V^{\mu} V_{\mu}-\frac{1}{4}\left|M_{i j}\right|^{2}+\left|\partial_{\mu} \Phi_{i}^{\alpha}+\frac{1}{2} V_{i \mu}^{k} \Phi_{k}^{\alpha}\right|^{2}\right)
\end{aligned}
$$

where $A_{\mu \nu}^{I}=F_{\mu \nu}^{I-}-\frac{1}{4} \bar{X}^{I} T_{\mu \nu}^{-}$with $F_{\mu \nu}^{I-}=\frac{1}{2}\left(F_{\mu \nu}^{I}-i^{*} F_{\mu \nu}^{I}\right)$. As we will see this particular combination plays an important role. Note that the fields are subject to the constraint

$$
\nabla^{\mu} V_{\mu}-\frac{1}{2} V^{\mu} V_{\mu}-\frac{1}{4}\left|M_{i j}\right|^{2}+\left|\partial_{\mu} \Phi_{i}^{\alpha}+\frac{1}{2} \mathcal{V}_{i \mu}^{k} \Phi_{k}^{\alpha}\right|^{2}=D-\frac{1}{3} R
$$

For more details and also the definition of other components which we have used here see [36]. 
Let us now consider an extremal black hole in this supergravity theory with near horizon geometry of the form [25]

$$
\begin{aligned}
d s^{2} & =v_{1}\left(-r^{2} d t^{2}+\frac{d r^{2}}{r^{2}}\right)+v_{2}\left(d \theta^{2}+\sin ^{2} \theta d \phi^{2}\right), \\
F_{r t}^{I} & =e^{I}, \quad F_{\theta \phi}^{I}=p^{I} \sin \theta, \quad X^{I}=x^{I}, \quad T_{r t}^{-}=v_{1} \omega .
\end{aligned}
$$

The other fields are given by

$$
\mathcal{A}_{\mu}=0, \quad \mathcal{V}_{j \mu}^{i}=0, \quad V_{\mu}=0, \quad M_{i j}=0, \quad Y_{i j}^{I}=0, \quad \Phi_{i}^{\alpha}=\delta_{i}^{\alpha}, \quad D-\frac{1}{3} R=0 .
$$

It is easy to see that this is a consistent truncation. Note that for the ansatz we are considering we have

$$
\hat{A}=-4 \omega^{2}, \quad A_{\mu \nu}^{I} \equiv v_{1} A^{I}=e^{I}-i \frac{v_{1}}{v_{2}} p^{I}-\frac{1}{2} \bar{x}^{I} v_{1} \omega
$$

Following [16] the entropy function is defined as

$$
\mathcal{E}\left(v_{1}, v_{2}, \omega, x^{I}, e^{I}, q_{I}, p^{I}\right)=2 \pi\left(-\frac{1}{2} q_{I} e^{I}-\int d \theta d \phi \sqrt{-G} \mathcal{L}\right)
$$

which for our ansatz it reads

$$
\begin{aligned}
\mathcal{E} & =-\pi q_{I} e^{I}-\pi v_{1} v_{2}\left\{i\left(v_{1}^{-1}-v_{2}^{-1}\right)\left(x^{I} \bar{F}_{I}-\bar{x}^{I} F_{I}\right)+\frac{i}{8}\left(\bar{\omega}^{2} F-\omega^{2} \bar{F}\right)\right. \\
& -\frac{i}{4} F_{I J} A^{I} A^{J}+\frac{i}{4} \bar{F}_{I J} \bar{A}^{I} \bar{A}^{J}-\frac{i}{4} \omega \bar{F}_{I} A^{I}+\frac{i}{4} \bar{\omega} F_{I} \bar{A}^{I} \\
& \left.+8 i \omega \bar{\omega}\left(-v_{1}^{-1}-v_{2}^{-1}+\frac{1}{8} \omega \bar{\omega}\right)\left(F_{\hat{A}}-\bar{F}_{\hat{A}}\right)+64 i\left(v_{1}^{-1}-v_{2}^{-1}\right)^{2}\left(F_{\hat{A}}-\bar{F}_{\hat{A}}\right)\right\} .
\end{aligned}
$$

In this framework the equations of motion can be obtained by extremizing the entropy function i.e.

$$
\frac{\partial \mathcal{E}}{\partial v_{i}}=0, \quad \frac{\partial \mathcal{E}}{\partial x^{I}}=0, \quad \frac{\partial \mathcal{E}}{\partial \omega}=0, \quad \frac{\partial \mathcal{E}}{\partial e^{I}}=0 .
$$

The entropy function defined here is invariant under local scale transformation

$$
x^{I} \rightarrow \lambda x^{I}, \quad v_{i} \rightarrow \lambda^{-1} \bar{\lambda}^{-1} v_{i}, \quad e^{I} \rightarrow e^{I}, \quad \omega \rightarrow \lambda \omega, \quad q_{I} \rightarrow q_{I}, \quad p^{I} \rightarrow p^{I} .
$$

This is related to the conformal symmetry of the $\mathcal{N}=2$ supergravity theory action. In special geometry one can fix this symmetry using the symplectic constraint on $\left(L^{I}, M_{I}\right)$ that is $i\left(\bar{L}^{I} M_{I}-L^{I} \bar{M}_{I}\right)=1$. In principle one should fix the gauge, though, it is more convenient to work with gauge invariant action. Later on we will fix the gauge in the level of equations of motion. We note, however, that the gauge can be 
fixed in several ways. In particular, following [25], the scaling symmetry in entropy function can be eliminated by imposing the condition

$$
\omega=\text { constant }
$$

on the equations of motion.

It is also worth noting that if we eliminate $e^{I}$, using the equations of motion for $e^{I}, \frac{\partial \mathcal{E}}{\partial e^{I}}=0$, one can see that entropy function is invariant under symplectic transformation which acts on $\left(p^{I}, q_{I}\right)$ and $\left(X^{I}, F_{I}\right)$ as follows:

$$
\left(\begin{array}{c}
\check{X}^{I} \\
\check{F}_{J}
\end{array}\right)=\left(\begin{array}{cc}
U_{K}^{I} & Z^{I L} \\
W_{J K} & V_{J}^{L}
\end{array}\right)\left(\begin{array}{c}
X^{K} \\
F_{L}
\end{array}\right), \quad\left(\begin{array}{c}
\check{p}^{I} \\
\check{q}_{J}
\end{array}\right)=\left(\begin{array}{cc}
U_{K}^{I} & Z^{I L} \\
W_{J K} & V_{J}^{L}
\end{array}\right)\left(\begin{array}{c}
p^{K} \\
q_{L}
\end{array}\right)
$$

where $U, Z, W$ and $V$ are each $(N+1) \times(N+1)$ matrices satisfying

$$
U^{T} W-W^{T} U=0, \quad Z^{T} V-V^{T} Z=0, \quad U^{T} V-W^{T} Z=1 .
$$

This symplectic invariance keeps other parameters unchanged. It should be mentioned that in entropy function formalism, the set $\left(X^{I}, F_{I}\right)$ plays the role of $\left(L^{I}, M_{I}\right)$ in special geometry.

Now we have all the ingredients we need to write the most general form of the attractor equations using the entropy function formalism. In fact the main purpose is to obtain the value of the scalars or moduli fields at the horizon in terms of the electric and magnetic charges of the black hole. Therefore as the first step one needs to extremize the entropy function with respect to $e^{I}$. Doing so, one gets

$$
q_{I}=i \frac{v_{2}}{4}\left[\left(\omega \bar{F}_{I}-\bar{\omega} F_{I}\right)+2\left(F_{I J} A^{J}-\bar{F}_{I J} \bar{A}^{J}\right)\right] .
$$

On the other hand taking the real and imaginary parts of $A^{I}$ one finds

$$
e^{I}=\frac{v_{1}}{4}\left[\left(\bar{\omega} x^{I}+\omega \bar{x}^{I}\right)+2\left(A^{I}+\bar{A}^{I}\right)\right]
$$

and

$$
p^{I}=i \frac{v_{2}}{4}\left[\left(\omega \bar{x}^{I}-\bar{\omega} x^{I}\right)+2\left(A^{I}-\bar{A}^{I}\right)\right] .
$$

The equations (3.15) and (3.17) are actually the generalized attractor equations for $\mathcal{N}=2$ supergravity in four dimensions where the higher order corrections have also been taken into account. In fact these equations should be compared to those in (2.11) which are the generalized attractor equations in leading order.

These equations can be applied to both BPS and non-BPS black hole solutions. Actually the supersymmetric and non-supersymmetric black holes correspond to the solutions with $A^{I}=0$ and $A^{I} \neq 0$, respectively. This is very similar to what we have in the special geometry framework of these theories where supersymmetric and 
non-supersymmetric solutions or attractor equations correspond to $D Z=\bar{D} \bar{Z}=0$ and $D Z \neq 0, \bar{D} \bar{Z} \neq 0$, respectively.

To understand the relation between supersymmetry and vanishing $A^{I}$ better, it is useful to look at the supersymmetry transformation of the spinor fields in the vector multiplet. In particular consider the variation of gaugini under supersymmetry transformation generated by $Q$ with the parameters $\epsilon_{i}$ in the notation of [36]

$$
\delta \Omega_{i}^{I}=2 \gamma^{\mu} D_{\mu} X^{I} \epsilon_{i}+\frac{1}{2} \epsilon_{i j} \gamma^{\mu} \gamma^{\nu} \mathcal{F}_{\mu \nu}^{I-} \epsilon^{j}+Y_{i j}^{I} \epsilon^{j}+2 X^{I} \eta_{i}
$$

where $D$ is the covariant derivative with respect to all superconformal transformations and

$$
\mathcal{F}_{\mu \nu}^{I-}=F_{\mu \nu}^{I-}-\frac{1}{4} \bar{X}^{I} T_{\mu \nu}^{-}+\text {fermionic term. }
$$

The last term in the gaugini transformation is because of the special superconformal transformation given by the parameters $\eta_{i}$.

In the ansatz we are considering here, the covariant derivative is just a simple derivative and since in our ansatz $X^{I}$ are constant therefore the first terms is zero. The last term is also zero because we have already fixed the conformal gauge. On the other hand since there is no non-zero fermion in the definition of $\mathcal{F}_{\mu \nu}^{I-}$, we arrive at

$$
\delta \Omega_{i}^{I}=\frac{1}{2} \epsilon_{i j} \gamma^{\mu} \gamma^{\nu} \mathcal{F}_{\mu \nu}^{I-} \epsilon^{j} \propto A^{I} \epsilon_{i j} \epsilon^{j} .
$$

So for $A^{I}=0$ we would expect to get a supersymmetric solution, while for $A^{I} \neq 0$ it would be non-supersymmetric.

As a conclusion we note that the attractor equations are given in terms of the scalar fields and the functions $A^{I}$. In order to find the value of the scalar fields in terms of the black hole charges one first needs to find $A^{I}$ in terms of the moduli fields. This can be done by extremizing the entropy function with respect to other parameters $v_{1}, v_{2}, x^{I}$ and $\omega$. Doing so, one finds

$$
\begin{aligned}
\frac{\partial \mathcal{E}}{\partial x^{K}}=0 & =4\left(\frac{1}{v_{1}}-\frac{1}{v_{2}}\right)\left(\bar{F}_{K}-\bar{x}^{I} F_{I K}\right)-F_{K I J} A^{I} A^{J}-\bar{\omega} \bar{F}_{K I} \bar{A}^{I} \\
& +\bar{\omega} F_{K I} \bar{A}^{I}+32 \omega \bar{\omega}\left(-v_{1}^{-1}-v_{2}^{-1}+\frac{1}{8} \omega \bar{\omega}\right) F_{\hat{A} K}+256\left(v_{1}^{-1}-v_{2}^{-1}\right)^{2} F_{\hat{A} K} \\
\frac{\partial \mathcal{E}}{\partial v_{1}}=0 & =-8 v_{2}^{-1} \bar{F}_{I} x^{I}+2 F_{I J} A^{I} A^{J}+4 i v_{2}^{-1} F_{I J} p^{I} A^{J}+2 \omega F_{I J} \bar{x}^{I} A^{J} \\
& +2 i v_{2}^{-1} \omega \bar{F}_{I} p^{I}+\omega^{2} \bar{F}_{I} \bar{x}^{I}+\bar{\omega}^{2} F+64 \omega \bar{\omega}\left(-v_{2}^{-1}+\frac{1}{8} \omega \bar{\omega}\right) F_{\hat{A}} \\
& -512\left(v_{1}^{-2}-v_{2}^{-2}\right) F_{\hat{A}}-c . c ., \\
\frac{\partial \mathcal{E}}{\partial v_{2}}=0 & =8 v_{1}^{-1} \bar{F}_{I} x^{I}-4 i v_{2}^{-1} F_{I J} p^{I} A^{J}-2 i v_{2}^{-1} \omega \bar{F}_{I} p^{I}-2 F_{I J} A^{I} A^{J}
\end{aligned}
$$




$$
\begin{aligned}
& -2 \omega \bar{F}_{I} A^{I}+\bar{\omega}^{2} F+64 \omega \bar{\omega}\left(-v_{1}^{-1}+\frac{1}{8} \omega \bar{\omega}\right) F_{\hat{A}}+512\left(v_{1}^{-2}-v_{2}^{-2}\right) F_{\hat{A}}-c . c ., \\
\frac{\partial \mathcal{E}}{\partial \omega}=0 & =2 \omega F_{I J \hat{A}} A^{I} A^{J}+\frac{1}{4} F_{I J} \bar{x}^{I} A^{J}-2 \bar{\omega} \omega F_{I \hat{A}} \bar{A}^{I}-\frac{1}{4} \bar{F}_{I} A^{I} \\
& +8 \omega\left(v_{1}^{-1}-v_{2}^{-1}\right) \bar{x}^{I} F_{I \hat{A}}+8 \bar{\omega}\left(-v_{1}^{-1}-v_{2}^{-1}+\frac{1}{8} \omega \bar{\omega}\right)\left(F_{\hat{A}}-\bar{F}_{\hat{A}}\right) \\
& -64 \omega F_{\hat{A} \hat{A}}\left(\omega \bar{\omega}\left(-v_{1}^{-1}-v_{2}^{-1}+\frac{1}{8} \omega \bar{\omega}\right)-8\left(v_{1}^{-1}-v_{2}^{-1}\right)^{2}\right) .
\end{aligned}
$$

These equations are enough to find $A^{I}, v_{1}$ and $v_{2}$ in terms of $x^{I}$. Then by plugging them into the attractor equations one can find the moduli $x^{I}$ in terms of the electric and magnetic charges of the black hole as expected from attractor behavior. Finally due to the entropy function formalism the entropy associated with the black hole is given by the value of the entropy function at the extremum

$$
S_{B H}=\left.\mathcal{E}\right|_{\text {extremum }}
$$

\subsection{Explicit example}

To see how these attractor equations work, let us consider a specific theory with three vector multiplets and a prepotential

$$
F\left(X^{0}, X^{1}, X^{2}, X^{3}, \hat{A}\right)=-\frac{X^{1} X^{2} X^{3}}{X^{0}}-C \hat{A} \frac{X^{1}}{X^{0}} .
$$

This is the theory known as STU model with the identification

$$
\frac{X^{1}}{X^{0}}=i S, \quad \frac{X^{2}}{X^{0}}=i T, \quad \frac{X^{3}}{X^{0}}=i U .
$$

which describes a subsector of the low energy effective action for tree level Heterotic string theory on $T^{4} \times T^{2}$ or $K_{3} \times T^{2}$. For such a prepotential, the equations of motion derived from the Lagrangian density are invariant under $S O(2,2)=S L(2, R) \times$ $S L(2, R)$ T-duality symmetry. If we define the electric and magnetic charges related to the gauge fields as

$$
\begin{aligned}
& q_{0}=Q_{4}, \quad q_{1}=P_{4}, \quad q_{2}=Q_{1}, \quad q_{3}=Q_{3}, \\
& p^{0}=P_{2}, \quad p^{1}=-Q_{2}, \quad p^{2}=P_{3}, \quad p^{3}=P_{1},
\end{aligned}
$$

we can easily see that under $S O(2,2)$ duality transformations, $\vec{Q}$ and $\vec{P}$ behave in a way that $Q^{2}, P^{2}$ and $Q . P$ given by

$$
Q^{2}=2\left(Q_{1} Q_{3}+Q_{2} Q_{4}\right), \quad P^{2}=2\left(P_{1} P_{3}+P_{2} P_{4}\right)
$$




$$
Q . P=\left(Q_{1} P_{3}+Q_{3} P_{1}+Q_{2} P_{4}+Q_{4} P_{2}\right),
$$

remain invariant. Due to this symmetry one has the freedom to work in a frame in which $p^{0}=0$. Therefore using the attractor equation for $p^{0}$ in (3.17), we choose $X^{0}$ to be real and as a result $A^{0}$ will also be real.

We can now proceed to solve the equations of motion for this case. For the moment we assume $C=0$, or in other words we consider the leading order term. Setting $x^{I}=y^{I}+i z^{I}$ with $z^{0}=0$, one can see that the most general solutions of the equations of motion are given by

$$
v_{1}=v_{2}=\frac{16}{\omega \bar{\omega}},
$$

and

$$
A^{I}=0,
$$

or

$$
A^{I}=-\frac{1}{4}\left(3 y^{I}+i z^{I}\right),
$$

which correspond to supersymmetric and non-supersymmetric solutions, respectively. Note that we have fixed our gauge by choosing $\omega=\frac{1}{2}$.

Let us consider the supersymmetric solution given by (3.28). Plugging this solution into the attractor equations given by (3.15) and (3.17) we can find the value of the moduli at the attractor points as follows

$$
\begin{aligned}
x^{0} & =-\frac{1}{16} Q_{2} \sqrt{\frac{P^{4}}{P^{2} Q^{2}-(P . Q)^{2}}}, \frac{x^{1}}{x^{0}}=-\frac{P . Q}{P^{2}}+i \sqrt{\frac{P^{2} Q^{2}-(P . Q)^{2}}{P^{4}}} \\
\frac{x^{2}}{x^{0}} & =-\frac{1}{2 Q_{2} P_{1}}\left(Q_{2} P_{4}+Q_{1} P_{3}-P_{1} Q_{3}\right)-i \frac{P_{3}}{Q_{2}} \sqrt{\frac{P^{2} Q^{2}-(P . Q)^{2}}{P^{4}}} \\
\frac{x^{3}}{x^{0}} & =-\frac{1}{2 Q_{2} P_{3}}\left(Q_{2} P_{4}-Q_{1} P_{3}+P_{1} Q_{3}\right)-i \frac{P_{1}}{Q_{2}} \sqrt{\frac{P^{2} Q^{2}-(P . Q)^{2}}{P^{4}}}
\end{aligned}
$$

This solution is physical for $P^{2}>0$ and $(Q . P)^{2}<Q^{2} P^{2}$. Using the entropy function evaluated at the extremum, the entropy of these supersymmetric black holes is

$$
S_{B H}=\frac{\pi}{2} \sqrt{P^{2} Q^{2}-(P \cdot Q)^{2}} .
$$

Similarly one can proceed to the other solution which is non-BPS, (3.29), to find the values of the moduli at the horizon

$$
\begin{aligned}
x^{0} & =-\frac{1}{32} Q_{2} \sqrt{\frac{P^{4}}{-P^{2} Q^{2}+(P . Q)^{2}}}, \frac{x^{1}}{x^{0}}=-\frac{P \cdot Q}{P^{2}}+i \sqrt{\frac{-P^{2} Q^{2}+(P . Q)^{2}}{P^{4}}}, \\
\frac{x^{2}}{x^{0}} & =-\frac{1}{2 Q_{2} P_{1}}\left(Q_{2} P_{4}+Q_{1} P_{3}-P_{1} Q_{3}\right)-i \frac{P_{3}}{Q_{2}} \sqrt{\frac{-P^{2} Q^{2}+(P . Q)^{2}}{P^{4}}}
\end{aligned}
$$




$$
\frac{x^{3}}{x^{0}}=-\frac{1}{2 Q_{2} P_{3}}\left(Q_{2} P_{4}-Q_{1} P_{3}+P_{1} Q_{3}\right)-i \frac{P_{1}}{Q_{2}} \sqrt{\frac{-P^{2} Q^{2}+(P \cdot Q)^{2}}{P^{4}}},
$$

which is the same as supersymmetric case, but with a minus sign in $P^{2} Q^{2}-(P . Q)^{2}$. This corresponds to the case where $P^{2}>0$ and $(Q . P)^{2}>Q^{2} P^{2}$. This is the nonsupersymmetric black hole solution with the entropy

$$
S_{B H}=\frac{\pi}{2} \sqrt{-P^{2} Q^{2}+(P \cdot Q)^{2}} .
$$

In the more simplified BPS case with only 4 charges non-zero which are given by

$$
P_{1}=P_{3}=P_{0}, \quad Q_{2}=Q_{4}=-Q_{0}, \quad Q_{1}=Q_{3}=P_{2}=P_{4}=0,
$$

it can be seen that the non-BPS solution can be obtained (up to a normalization in our notation) by canonical transformation on BPS solution [37] which is

$$
P_{1}=P_{3}=P_{0}, \quad-Q_{2}=Q_{4}=-Q_{0}, \quad Q_{1}=Q_{3}=P_{2}=P_{4}=0 .
$$

This canonical transformation in leading order preserves the effective potential and entropy of the black hole. We note, however, that it is not obvious if higher order corrections would respect this canonical transformation.

The next step is to consider higher order corrections which correspond to the case where $C \neq 0$. To do this one needs to solve the equations with $C \neq 0$. Since these equations are algebraic equations in principle one can solve them. In particular for the supersymmetric case doing so, in the gauge of $\omega=\frac{1}{2}$, one finds [25]

$$
\begin{aligned}
x^{0} & =-\frac{1}{16} Q_{2} \sqrt{\frac{P^{2}\left(P^{2}+512 C\right)}{P^{2} Q^{2}-(P . Q)^{2}}}, \frac{x^{1}}{x^{0}}=-\frac{P . Q}{P^{2}}+i \sqrt{\frac{P^{2} Q^{2}-(P . Q)^{2}}{P^{2}\left(P^{2}+512 C\right)}}, \\
\frac{x^{2}}{x^{0}} & =-\frac{1}{2 Q_{2} P_{1}}\left(Q_{2} P_{4}+Q_{1} P_{3}-P_{1} Q_{3}\right)-i \frac{P_{3}}{Q_{2}} \sqrt{\frac{P^{2} Q^{2}-(P . Q)^{2}}{P^{2}\left(P^{2}+512 C\right)}}, \\
\frac{x^{3}}{x^{0}} & =-\frac{1}{2 Q_{2} P_{3}}\left(Q_{2} P_{4}-Q_{1} P_{3}+P_{1} Q_{3}\right)-i \frac{P_{1}}{Q_{2}} \sqrt{\frac{P^{2} Q^{2}-(P . Q)^{2}}{P^{2}\left(P^{2}+512 C\right)}},
\end{aligned}
$$

with $v_{1}=v_{2}=64$ and $A^{I}=0$. The entropy is given by

$$
S_{\mathrm{BH}}=\frac{\pi}{2} \sqrt{P^{2} Q^{2}-(P . Q)^{2}} \sqrt{1+\frac{512 C}{P^{2}}} .
$$

To compare this with the results given in terms of the special geometry $[18,26]$ it is instructive to rewrite the corrected entropy in terms of the prepotential. From the entropy function one gets

$$
S_{\mathrm{BH}}=2 \pi\left(-\frac{1}{2} q_{I} e^{I}-16 i\left(\omega^{-2} F-\bar{\omega}^{-2} \bar{F}\right)\right) .
$$


By making use of the attractor equations $q_{I}=4 i\left(\bar{\omega}^{-1} \bar{F}_{I}-\omega^{-1} F_{I}\right)$ and $e^{I}=4\left(\bar{\omega}^{-1} \bar{x}^{I}+\right.$ $\left.\omega^{-1} x^{I}\right)$ the entropy reads

$$
S_{\mathrm{BH}}=\pi\left(8\left(p^{I} F_{I}-q_{I} x^{I}\right)-256 \operatorname{Im}\left(F_{\hat{A}}\right)\right),
$$

which is the same as that obtained in $[18,26]$.

\section{Black hole partition function}

In the previous section we have studied the generalized attractor equations in presence of higher order corrections using entropy function formalism. The aim of this section is to use this formalism to understand the physical interpretation of these equations better. To do this we compare the entropy function formalism to [1]. In the following we shall review the relevant part of the paper.

The attractor equations for BPS black hole in $\mathcal{N}=2$ four dimensional supergravity could be used to express the entropy of the extremal black holes as the Legendre transformation of a function, $\mathcal{F}$, which is given by the imaginary part of the prepotential evaluated at the attractor point

$$
S_{\mathrm{BH}}(\vec{p}, \vec{q})=\mathcal{F}(\vec{e}, \vec{p})-e^{I} \frac{\partial \mathcal{F}(\vec{e}, \vec{p})}{\partial e^{I}} \equiv \mathcal{F}(\vec{e}, \vec{p})-e^{I} q_{I}
$$

where $(\vec{q}, \vec{p})$ are electric and magnetic charges of the black hole and $e$ is the electric potential defined by $e^{I}=-\frac{\partial S_{\mathrm{BH}}}{\partial q_{I}}$. It is natural to define a mixed partition function for black hole as follows

$$
Z_{\mathrm{BH}}(\vec{e}, \vec{p})=\sum_{\vec{q}} d(\vec{p}, \vec{q}) e^{\vec{e} \cdot \vec{q}}
$$

Here $d(\vec{p}, \vec{q})$ is integer black hole degeneracy and $\ln d(\vec{p}, \vec{q})$ is the microcanonical entropy. Therefore one leads to the following expression for the black hole partition function in terms of the function $\mathcal{F} 4$

$$
Z_{\mathrm{BH}}(\vec{p}, \vec{e})=e^{\mathcal{F}(\vec{p}, \vec{e})}
$$

Since in the entropy function formalism one gets the attractor equations from the equations of motion, it is natural to ask if we can follow the above procedure to define a (mixed) partition function using the entropy function formalism.

Form entropy function formalism we learned that the entropy of the extremal black hole is given by

$$
S_{\mathrm{BH}}\left(p^{I}, q_{I}\right)=2 \pi\left(e^{I} \frac{\partial f}{\partial e^{I}}-f\right)=2 \pi\left(e^{I} q_{I}-f\right) .
$$

\footnotetext{
${ }^{4}$ Since it is a mixed partition function, a priori it is not clear whether one can interpret $\mathcal{F}$ as the black hole free energy. We would like to thank C. Vafa for a discussion on this point.
} 
Comparing the Wald formula in the form of (4.4) and the fact that the equations of motion would lead to the attractor equations, it is tempting to follow OSV proposal to define a partition function for the corresponding extremal black hole as $Z_{\mathrm{BH}}=e^{-f}$.

Let us apply the above procedure to a toy model given by the following action

$$
S=\frac{1}{16 \pi} \int d^{4} x \sqrt{-G}\left(R-F^{2}\right) .
$$

Consider an extremal black hole solution with near horizon geometry given by

$$
d s^{2}=v_{1}\left(-r^{2} d t^{2}+\frac{d r^{2}}{r^{2}}\right)+v_{2}\left(d \theta^{2}+\sin ^{2} \theta d \phi^{2}\right), \quad F_{r t}=e .
$$

So that $f\left(v_{1}, v_{2}, e\right)=\frac{1}{2} v_{1} v_{2}\left(\frac{v_{1}-v_{2}}{v_{1} v_{2}}+\frac{e^{2}}{v_{1}^{2}}\right)$. Extremizing $f$ with respect to $v_{1}, v_{2}$ we get $v_{1}=v_{2}=e^{2}$. On the other hand we have $q=\frac{\partial f}{\partial e}=e$ and therefore from (4.4) we find $S_{\mathrm{BH}}=\pi q^{2}$.

Alternatively, using the fact that $f=e^{2} / 2$ one can define a partition function as $Z(e)=e^{-e^{2} / 2}$ and therefore we find the microscopic degrees of freedom as follows

$$
d(q)=\int d e e^{2 \pi\left(q e-\frac{1}{2} e^{2}\right)}=e^{\pi q^{2}} \Rightarrow S_{\text {micro }}=\ln d(q)=\pi q^{2}
$$

in agreement with the black hole entropy. Therefore we can conclude that the entropy of the black hole is exactly given by the microcanonical entropy. It is also possible to consider higher order corrections to the action. In the present case the corrections can be given by the Gauss-Bonnet action which leads to a correction as $d(q)=e^{\pi q^{2}+2 \pi \lambda}$ or $S_{\text {micro }}=\pi q^{2}+2 \pi \lambda$, in agreement with the Wald formula for the black hole entropy in the presence of the Gauss-Bonnet term [19]. Here $\lambda$ is the coefficient of the Gauss-Bonnet term.

To be more realistic we consider the system we have studied in the previous section. By making use of the results in the previous section one finds

$$
\begin{aligned}
f\left(v_{1}, v_{2}, e^{I}, p^{I}, x^{I}, \omega\right) & =\frac{1}{2} v_{1} v_{2}\left\{i\left(v_{1}^{-1}-v_{2}^{-1}\right) x^{I} \bar{F}_{I}-\frac{i}{4} F_{I J} A^{I} A^{J}-\frac{i}{4} \omega \bar{F}_{I} A^{I}\right. \\
& +\left(8 i \omega \bar{\omega}\left(-v_{1}^{-1}-v_{2}^{-1}+\frac{1}{8} \omega \bar{\omega}\right)+64 i\left(v_{1}^{-1}-v_{2}^{-1}\right)^{2}\right) F_{\hat{A}} \\
& \left.+\frac{i}{8} \bar{\omega}^{2} F+\text { c.c. }\right\} .
\end{aligned}
$$

In the supersymmetric case from equations (3.21) we find $A^{I}=0$ and $v_{1}=v_{2}=\frac{16}{\omega \bar{\omega}}$. So one arrives at

$$
f\left(e^{I}, p^{I}\right)=-2 \operatorname{Im}\left(\left(\frac{4}{\omega}\right)^{2} F\left(x^{I}, \omega\right)\right),
$$


where $x^{I}=\frac{\omega}{8}\left(e^{I}+i p^{I}\right)$. It is worth noting that in comparison with [1] $f$ can be identified with $-\mathcal{F}$ and therefore following [1] the system can be described as a mixed ensemble.

Since, in general, the equations (3.21) have two solutions, supersymmetric and non-supersymmetric, one might naturally expect that the situation would also go through the non-supersymmetric case. In fact from the equations of motion one may first obtain $A^{I}$ and therefore using the relation $\bar{A}^{I}+\frac{\bar{\omega}}{2} x^{I}=v_{1}^{-1}\left(e^{I}+i v_{1} v_{2}^{-1} p^{I}\right)$ we can find the moduli in terms of $e^{I}$ and $p^{I}$. Plugging the results into (4.8) one finds $f$ as a function of $e^{I}$ and $p^{I}$ and thereby the partition function can be evaluated along the supersymmetric case. Partition function for non-BPS solution has also been studied in $[38,39]$.

Regarding the fact that the entropy function formalism could simply reproduce the known results for BPS case and also is powerful enough to be generalized to nonBPS solution, one then might naively think that we can generalize it for theories without supersymmetry as well. We note, however, that it is not obvious whether this is going to be the case. In fact a priori it is not clear if the black hole could be described by a mixed ensemble even though the entropy is given by a Legendre transformation of the function $f$. We will come back to this point in the next section.

\section{Discussions}

In this paper we have shown how the entropy function can reproduce the generalized attractor equations and also how to generalize them while higher order corrections are also taken into account. In fact in the supersymmetric model we have studied in this paper we showed that one of the attractor equations comes out as the equation of motion and the other comes as the supersymmetry condition.

Having had the attractor equations in general form we have also tried to see if this can help us to define a partition function for the extremal black hole following [1]. In particular we have considered a particular example which is an extremal black hole in four dimensional supergravity obtained by compactification of type IIA on Calabi-Yau 3-fold. In this example we could identify the function $f$ with $-\mathcal{F}$ and thereby to define a mixed partition function as $e^{-f}$.

We note, however, that these black holes can also be studied from Heterotic string point of view. It is then natural to ask if this description also leads to the same conclusion. Therefore it is worth to reconsider the model from the Heterotic string point of view. To do this we shall restrict ourselves to two charged black hole, though the generalization for higher charges is straightforward.

For two charged black hole it is known that in leading order the entropy is zero while higher order corrections (Gauss-Bonnet in the present case) stretches the horizon leading to non-zero entropy. Taking into account the Gauss-Bonnet term and doing the same computations as that in the previous section (see also [20]) we find $f=0$. Therefore it seems impossible to use the OSV procedure to define a 
(mixed) partition function. In fact there is a proposal for the statistical ensemble one may associate to this black hole due to Sen [41] who conjectured that, after taking into account the holomorphic anomaly, a grand canonical ensemble underlies the system. On the other hand from our considerations in the previous section which has been done in the type IIA dual description we have been able to get a mixed partition function using entropy function mechanism (see also [28] where the author has considered the system as a mixed ensemble and confirmed OSV conjecture without taking into account the holomorphic anomaly term.).

Therefore we face a puzzle, namely, studying the system from two different point of views, leads to two different ensembles. So far we do not have a good interpretation of this observation. As far as the technical point is concerned we note that changing type IIA description to Heterotic description we have lost one of the attractor equations. As we have mentioned one of the equations comes from the equations of motion while the other one is the condition we get from supersymmetric condition which as we have seen, depends on the way we incorporate the supersymmetry in the theory.

We also note that it might be related to the fact that OSV proposal is not symplectic invariant. In particular it begins with a symplectic invariant answer ( Wald formula) and performs a Legendre transform and an inverse Laplace transform both of which are non-symplectic invariant operation. It is not guaranteed that the final answer will be symplectic invariant. In fact the entropy function seems to be a more natural object than the Lagrangian density $f$, (the latter is not symplectic invariant while the former is) 5 .

As a final remark we note that the basic point in entropy function formalism is the fact that near horizon field configuration of these extremal black holes is fixed just by using the symmetries of near horizon geometry that is $A d S_{2} \times S^{2}$. This is, in fact, the notion of attractor mechanism which means that the value of the scalar fields at the horizon are independent of their values at infinity and they are fixed by the black hole charges. Moreover the entropy of the black hole is just given by the black hole charges too. Therefore one may conclude that the entropy function formalism leads us to the fact that the near horizon field configuration has enough information about the corresponding extremal black hole. In this sense, in comparison with AdS/CFT [42-44], one might suspect that the attractor behavior plays the role of the decoupling limit in this context.

It is worth noting that the black hole attractor mechanism can also be treated as the holographically dual to a conformally invariant quantum mechanics [47]. This might also indicate that the near horizon modes have enough information about the whole system. It would be very interesting to understand this connection better. To do this, it might be useful to consider the supersymmetric case where one may use the results of $[45,46]$. In this supersymmetric case where we consider type IIA string theory compactified on Calabi-Yau 3-fold the four dimensional theory may have an

\footnotetext{
${ }^{5}$ We would like to thank A. Sen for a discussion on this point.
} 
extremal black hole solution which can be studied using the $A d S_{2} \times S^{2}$ background. In this case the flux data on the $A d S_{2} \times S^{2}$ geometry is mapped to the charges of the dual black hole and its entropy is the logarithm of the norm of the Hartle-Hawking wave function on $A d S_{2} \times S^{2}[45,46]$.

\section{Acknowledgments}

We would like to thank Ashoke Sen, Alireza Tavanfar and Cumrun Vafa for useful discussions and comments.

\section{References}

[1] H. Ooguri, A. Strominger and C. Vafa, "Black hole attractors and the topological string," Phys. Rev. D 70, 106007 (2004) arXiv:hep-th/0405146.

[2] S. Ferrara, R. Kallosh and A. Strominger, "N=2 extremal black holes," Phys. Rev. D 52, 5412 (1995) arXiv:hep-th/9508072].

[3] A. Strominger, "Macroscopic Entropy of $N=2$ Extremal Black Holes," Phys. Lett. B 383, 39 (1996) arXiv:hep-th/9602111.

[4] S. Ferrara and R. Kallosh, "Supersymmetry and Attractors," Phys. Rev. D 54, 1514 (1996) arXiv:hep-th/9602136.

[5] S. Ferrara and R. Kallosh, "Universality of Supersymmetric Attractors," Phys. Rev. D 54, 1525 (1996) |arXiv:hep-th/9603090].

[6] S. Ferrara, G. W. Gibbons and R. Kallosh, "Black holes and critical points in moduli space," Nucl. Phys. B 500, 75 (1997) arXiv:hep-th/9702103.

[7] G. W. Gibbons, R. Kallosh and B. Kol, "Moduli, scalar charges, and the first law of black hole thermodynamics," Phys. Rev. Lett. 77, 4992 (1996) arXiv:hep-th/9607108.

[8] P. K. Tripathy and S. P. Trivedi, "Non-supersymmetric attractors in string theory," arXiv:hep-th/0511117.

[9] R. Kallosh, "New attractors," arXiv:hep-th/0510024.

[10] A. Giryavets, "New attractors and area codes," arXiv:hep-th/0511215.

[11] G. Dall'Agata, "Non-Kaehler attracting manifolds," arXiv:hep-th/0602045.

[12] R. Kallosh, N. Sivanandam and M. Soroush, "The non-BPS black hole attractor equation," arXiv:hep-th/0602005.

[13] S. Bellucci, S. Ferrara and A. Marrani, "On some properties of the Attractor Equations," arXiv:hep-th/0602161. 
[14] K. Goldstein, N. Iizuka, R. P. Jena and S. P. Trivedi, "Non-supersymmetric attractors," arXiv:hep-th/0507096.

[15] B. Chandrasekhar, S. Parvizi, A. Tavanfar and H. Yavartanoo, "Nonsupersymmetric attractors in $\mathrm{R}^{* *} 2$ gravities," arXiv:hep-th/0602022.

[16] A. Sen, "Black hole entropy function and the attractor mechanism in higher derivative gravity," JHEP 0509, 038 (2005) arXiv:hep-th/0506177).

[17] K. Behrndt, G. Lopes Cardoso, B. de Wit, R. Kallosh, D. Lust and T. Mohaupt, "Classical and quantum N = 2 supersymmetric black holes," Nucl. Phys. B 488, 236 (1997) arXiv:hep-th/9610105.

[18] T. Mohaupt, "Strings, higher curvature corrections, and black holes," arXiv:hep-th/0512048.

[19] M. Alishahiha and H. Ebrahim, "Non-supersymmetric attractors and entropy function," JHEP 0603, 003 (2006) [arXiv:hep-th/0601016].

[20] A. Sen, "Entropy function for heterotic black holes," arXiv:hep-th/0508042.

[21] P. Prester, "Lovelock type gravity and small black holes in heterotic string theory," JHEP 0602, 039 (2006) arXiv:hep-th/0511306.

[22] A. Sinha and N. V. Suryanarayana, "Extremal single-charge small black holes: Entropy function analysis," Class. Quant. Grav. 23, 3305 (2006) arXiv:hep-th/0601183.

[23] B. Chandrasekhar, "Born-Infeld corrections to the entropy function of heterotic black holes," arXiv:hep-th/0604028.

[24] A. Ghodsi, " $R^{4}$ corrections to D1D5p black hole entropy from entropy function arXiv:hep-th/0604106.

[25] B. Sahoo and A. Sen, "Higher derivative corrections to non-supersymmetric extremal black holes in $\mathcal{N}=2$ supergravity," arXiv:hep-th/0603149.

[26] G. Lopes Cardoso, B. de Wit and T. Mohaupt, "Corrections to macroscopic supersymmetric black-hole entropy," Phys. Lett. B 451, 309 (1999) arXiv:hep-th/9812082.

[27] G. Lopes Cardoso, B. de Wit, J. Kappeli and T. Mohaupt, "Black hole partition functions and duality," JHEP 0603, 074 (2006) arXiv:hep-th/0601108.

[28] A. Dabholkar, "Exact counting of black hole microstates," Phys. Rev. Lett. 94, 241301 (2005) arXiv:hep-th/0409148. 
[29] A. Dabholkar, F. Denef, G. W. Moore and B. Pioline, "Exact and asymptotic degeneracies of small black holes," JHEP 0508, 021 (2005) arXiv:hep-th/0502157.

[30] A. Dabholkar, F. Denef, G. W. Moore and B. Pioline, "Precision counting of small black holes," JHEP 0510, 096 (2005) arXiv:hep-th/0507014.

[31] S. Parvizi and A. Tavanfar, "Minimal redefinition of the OSV ensemble," arXiv:hep-th/0508231.

[32] F. Denef, "Supergravity flows and D-brane stability," JHEP 0008, 050 (2000) arXiv:hep-th/0005049.

[33] B. de Wit, J. W. van Holten and A. Van Proeyen, "Transformation Rules Of N=2 Supergravity Multiplets," Nucl. Phys. B 167, 186 (1980).

[34] B. de Wit, J. W. van Holten and A. Van Proeyen, "Structure Of N=2 Supergravity," Nucl. Phys. B 184, 77 (1981) [Erratum-ibid. B 222, 516 (1983)].

[35] B. de Wit, P. G. Lauwers, R. Philippe, S. Q. Su and A. Van Proeyen, "Gauge And Matter Fields Coupled To N=2 Supergravity," Phys. Lett. B 134, 37 (1984).

[36] T. Mohaupt, "Black hole entropy, special geometry and strings," Fortsch. Phys. 49, 3 (2001) arXiv:hep-th/0007195].

[37] R. Kallosh, "From BPS to non-BPS black holes canonically," arXiv:hep-th/0603003.

[38] S. Parvizi and A. Tavanfar, "Partition function of non-supersymmetric black holes in the supergravity limit," arXiv:hep-th/0602292.

[39] S. Ferrara and R. Kallosh, "On N = 8 attractors," arXiv:hep-th/0603247.

[40] A. Sen, "Stretching the horizon of a higher dimensional small black hole," JHEP 0507, 073 (2005) arXiv:hep-th/0505122.

[41] A. Sen, "How does a fundamental string stretch its horizon?," JHEP 0505, 059 (2005) arXiv:hep-th/0411255.

[42] J. M. Maldacena, "The large N limit of superconformal field theories and supergravity," Adv. Theor. Math. Phys. 2, 231 (1998) [Int. J. Theor. Phys. 38, 1113 (1999)] arXiv:hep-th/9711200.

[43] S. S. Gubser, I. R. Klebanov and A. M. Polyakov, "Gauge theory correlators from non-critical string theory," Phys. Lett. B 428, 105 (1998) arXiv:hep-th/9802109. 
[44] E. Witten, "Anti-de Sitter space and holography," Adv. Theor. Math. Phys. 2, 253 (1998) arXiv:hep-th/9802150.

[45] H. Ooguri, C. Vafa and E. P. Verlinde, "Hartle-Hawking wave-function for flux compactifications," Lett. Math. Phys. 74, 311 (2005) arXiv:hep-th/0502211.

[46] S. Gukov, K. Saraikin and C. Vafa, "The entropic principle and asymptotic freedom," Phys. Rev. D 73, 066010 (2006) arXiv:hep-th/0509109].

[47] D. Gaiotto, A. Strominger and X. Yin, "Superconformal black hole quantum mechanics," JHEP 0511, 017 (2005) arXiv:hep-th/0412322. 